4) Postfix in one percent osmium tetroxide in buffer for 15 minutes.

$|0|$

We appreciate the response to this publication feature - and welcome all contributions. Contributions may be sent to Phil Oshel, our Technical Editor at:

Mr. Phil Oshel Tel:: (608)833-2885

POBox 620068 Fax: (608)836-1969

Middleton WI53562 eMail: peoshel@facstaff.wisc.edu

\section{HMDS Preparation of Cell Monolayers Grown on Thermanox Coverslips for SEM}

The use of hexamethyldisilazane (HMDS) is a convenient alternative to critical point drying for the preparation of cell monolayers grown on Thermanox cover slips for SEM.

1) Use 13 or $15 \mathrm{~mm}$ sized coverslips, which fit nicely into 24 well culture plates. For easier handling, cut two snips in the edge of the coverslip and bend up a tab to grasp from above with forceps. Sterilize with $70 \%$ ethanol.

2) Fix monolayers in $2.5 \%$ glutaraldehyde in $0.1 \mathrm{M}$ Sorensen's buffer for 30 minutes.

3) Rinse twice with buffer.



"The Evactron device can significantly reduce contamination in the SEM."

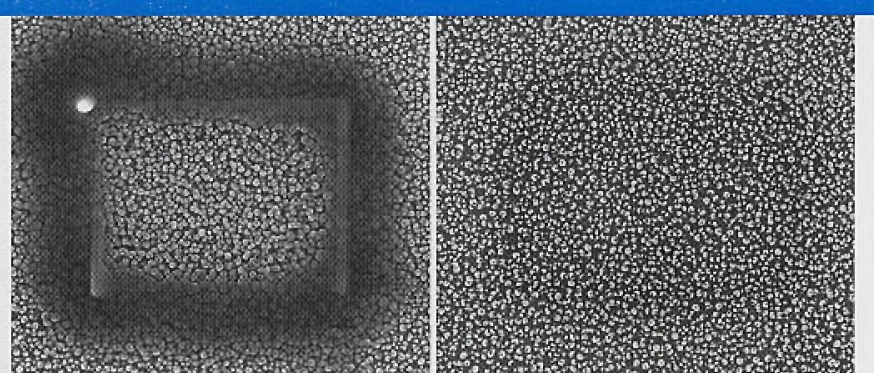

A silicon "grass" sample irradiated for 10 minutes before (left) and after (right) the use of Evactron SEM-CLEAN device. $50 \mathrm{kX}$ From Active Monitoring and Control of Electron Beam Induced Contamination by A. Vladar, M. Postek, \& R. Vane., SPIE Microlithography Conference. Feb. 2001.

\section{EVACTRON SEM-CLEAN "plasma" Cleaning \\ X E I scientific Anti-Contamination Systems for SEMs \\ 3124 Wessex Way, Redwood City, CA 94061 (650) 369-0133, FAX (650) 363-1659 email: RVaneXEI@concentric.net www.SEMCLEAN.COM}

5) Rinse twice with double distilled water.

6) Treat with one percent tannic acid for 20 minutes

7) Rinse with double distilled water.

8) Dehydrate in a graded series of ethanol, ending with thisee changes of $100 \%$ ethanol.

9) Infiltrate with four, five minute changes of full strength HMDS. (A gradual transition is unnecessary.)

10) Draw off all but a thin film of HMDS and leave the sampes in the hood overnight to off-gas.

11) The next day, transfer to a vacuum desiccator for fur off-gassing.

12) Affix to SEM stubs with double stick tape

13) Dot the edges of the coverslips with colloidal graphit: to bleed off charging.

14) Sputtercoat with gold

Dorothy Roak Sorenson, Univ. of Michigan Medical Schoôl dsoren@umich.edu

\section{Cold Vs. Schottky FE Guns}

The advantages/disadvantages of cold vs. Schottky FE guns lie at the extremes of performance. Cold FE will give you better ultimate image resolution (roughly 1.5 vs. $2.5 \mathrm{~nm}$, depending on manufacturer, of course) and will give you noticea bly better images at low voltage ( $1 \mathrm{kV}$ or so). Schottky will giv you much higher currents at larger spot sizes (for $\mathrm{x}$-ray mapt ping or back-scatter diffraction pattern acquisition, for exam ple) and much better medium-term (tens of minutes to a few 市 hours) beam current stability, if that is important to you. It is also not necessary to flash the tip periodically in a Schottky gun. For most other SEM needs, either form will work wonderfully for you.

We have one of each, and we do not notice any difference in the maintenance needs between them. Don't worry about tip replacements. Our cold FE is nearly 6 years old and is still running with the original tip, as is our Schottky, which is over three years old. I don't think this is a function of vendor - it is my impression that microscopes from other vendors have similar records. The tip replacement is performed by the service engineers, and depending on your service contract, may be included in that. It takes about 3 days (I am told!) on either instrument.

It is important to be clear about your microscopy needs regarding "variable pressure". There is a big difference between the ESEM of FEI/Philips and the variable pressure designs of other manufacturers, so one needs to be clear about how important this factor is to you. What structures will you need to examine by "conventional" SEM, how important is high-speed $x$-ray mapping, etc. etc. etc. By asking these kinds of questions the right microscope will eventually become clear to you. And one should not worry about the mechanics of the gun - they all work fine.

All in my humble opinion, of course!!

Tony Garratt-Reed, Massachusetts Inst. Of Technology tonygr@mit.edu

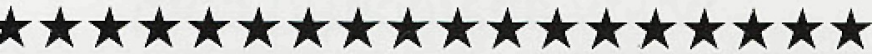

